PROCEEDINGS OF THE

AMERICAN MATHEMATICAL SOCIETY

Volume 125, Number 1, January 1997, Pages 87-92

S 0002-9939(97)03430-8

\title{
FACTORISATION IN NEST ALGEBRAS
}

\author{
M. ANOUSSIS AND E. G. KATSOULIS \\ (Communicated by Palle E. T. Jorgensen)
}

\begin{abstract}
We give a necessary and sufficient condition on an operator $A$ for the existence of an operator $B$ in the nest algebra $\operatorname{Alg} N$ of a continuous nest $N$ satisfying $A A^{*}=B B^{*}\left(\right.$ resp. $\left.A^{*} A=B^{*} B\right)$. We also characterise the operators $A$ in $B(H)$ which have the following property: For every continuous nest $N$ there exists an operator $B_{N}$ in $\operatorname{Alg} N$ satisfying $A A^{*}=B_{N} B_{N}^{*}$ (resp. $\left.A^{*} A=B_{N}^{*} B_{N}\right)$.
\end{abstract}

\section{INTRODUCTION-PRELIMINARIES}

The problem of factorisation of operators with respect to a nest algebra has been studied by many authors [8], [1], [13], [9], [11], [12], [10]. In this work we give a necessary and sufficient condition on an operator $A$ for the existence of an operator $B$ in the nest algebra $\operatorname{Alg} N$ of a continuous nest $N$ satisfying $A A^{*}=B B^{*}$ (resp. $A^{*} A=B^{*} B$ ). This result improves Theorem 4.9 in [9] for continuous nests. We also characterise the operators $A$ in $B(H)$ which have the following property: For every continuous nest $N$ there exists an operator $B_{N}$ in $\operatorname{Alg} N$ satisfying $A A^{*}=B_{N} B_{N}^{*}$ (resp. $\left.A^{*} A=B_{N}^{*} B_{N}\right)$.

Throughout this work $H$ denotes a separable Hilbert space and $B(H)$ the space of all bounded operators from $H$ into itself. If $V$ is a subset of $H$ we denote by $[V]$ the linear span of $V$. By subspace of $H$ we mean a subset of $H$ which is closed under addition of vectors and scalar multiplication. If $\left\{V_{n}\right\}_{n=1}^{\infty}$ is a sequence of closed mutually orthogonal subspaces of $H$ we denote by $\sum_{n=1}^{\infty} \oplus V_{n}$ the closure of their linear span. If $A$ is in $B(H)$ we denote by $r(A)$ the range of $A$ and by coker $A$ the orthogonal complement of the kernel of $A$. An operator range is the range of a bounded operator in $H$. A nest in $H$ is a totally ordered set of closed subspaces of $H$ containing $\{0\}$ and $H$ which is closed under intersection and closed span. If $N$ is a nest in $H$ and $P$ is in $N$ we will denote by the same symbol the orthogonal projection on the subspace $P$. If $N$ is a nest we denote by $N^{\perp}$ the nest $\left\{P^{\perp}: P \in N\right\}$. A nest $N$ is continuous if $P=\overline{\left[\bigcup_{Q<P} Q\right]}$ for every $P$ in $N$. Given a nest $N$ the associated nest algebra $\operatorname{Alg} N$ is the set of operators $A$ in $B(H)$ satisfying $P A P=A P$ for every $P$ in $N$. For a general discussion of nest algebras the reader is referred to [3].

Received by the editors December 6, 1994 and, in revised form, April 5, 1995.

1991 Mathematics Subject Classification. Primary 47D25.

(C)1997 American Mathematical Society 


\section{Proper SUBSpaces}

We introduce in this section the notion of $N$-proper subspace for a nest $N$. We show that a closed subspace of $H$ of co-finite dimension is $N$-proper for every continuous nest $N$.

Definition 1. Let $N$ be a nest on $H$. A vector $x$ in $H$ is called $N$-proper if $x=P x$ for some $P$ in $N, P \neq I$.

Definition 2. Let $N$ be a nest on $H$. A subspace $V$ of $H$ is called $N$-proper if $[V \cap P: P \in N, P \neq I]$ is dense in $V$.

Lemma 3. Let $N$ be a continuous nest on $H$. Let $\left\{P_{n}\right\}_{n=1}^{\infty}$ be a sequence of elements of $N$ such that: $P_{n} \neq I, P_{n+1} \geq P_{n}$, and $P_{n}$ converges strongly to $I$. Let $x_{1}, x_{2}, \ldots, x_{m}$ be orthonormal vectors in $H$. Set $V=\left[x_{1}, x_{2}, \ldots, x_{m}\right]^{\perp}$. Then:

(a) There exists $n_{0}$ such that $P_{n} x_{1}, P_{n} x_{2}, \ldots, P_{n} x_{m}$ are linearly independent for $n \geq n_{0}$.

(b) We set $V_{1}=P_{1} H \ominus P_{1} V^{\perp}$ and we define inductively

$$
V_{n}=P_{n} H \ominus\left(\sum_{i=1}^{n-1} \oplus V_{i} \oplus P_{n} V^{\perp}\right) .
$$

Then $V=\sum_{i=1}^{\infty} \oplus V_{i}$.

Proof. (a) The Grammian of the vectors $P_{n} x_{1}, P_{n} x_{2}, \ldots, P_{n} x_{m}$ converges to the Grammian of the vectors $x_{1}, x_{2}, \ldots, x_{m}$ which equals 1 .

(b) It is easy to see that the $V_{n}$ 's are mutually orthogonal and that $V_{n}$ is contained in $V$ for every $n$. We show that $\left(\sum_{i=1}^{\infty} \oplus V_{i}\right) \oplus V^{\perp}=H$. Let $x$ be a vector in $H$ which is orthogonal to $\left(\sum_{i=1}^{\infty} \oplus V_{i}\right) \oplus V^{\perp}$. For each $n$ the vector $P_{n} x$ is orthogonal to $\sum_{i=1}^{n} \oplus V_{i}$ so $P_{n} x$ is in $P_{n} V^{\perp}$. For $n \geq n_{0}$ we have $P_{n} x=P_{n}\left(\sum_{i=1}^{m} a_{i} x_{i}\right)$, where the $a_{i}$ 's are complex numbers not depending on $n$. So $x=\lim _{n \rightarrow \infty} P_{n} x=\sum_{i=1}^{m} a_{i} x_{i}$. But $x$ is orthogonal to $V^{\perp}$, hence it is 0 .

Proposition 4. Let $N$ be a continuous nest and $V$ a closed subspace of $H$ of cofinite dimension. Then $V$ is $N$-proper.

Proof. It follows immediately from Lemma 3.

Let $N$ be a continuous nest on $H$ and $A$ an operator in $B(H)$. Consider the set $\bigcup_{P \in N, P \neq I} A^{-1}(P)$. This set is equal to $\bigcup_{P \in N, P \neq I} \operatorname{Ker}\left(P^{\perp} A\right)$. If $A$ is an $\operatorname{Alg} N$ the set $\bigcup_{P \in N, P \neq I} A^{-1}(P)$ contains $\bigcup_{P \in N, P \neq I} P$; hence it is dense in $H$. There exist operators $A$ in $B(H)$ for which $\bigcup_{P \in N, P \neq I} A^{-1}(P)$ is not dense in $H$. We construct such an operator in Example 9. We will prove in the next section that $\bigcup_{P \in N, P \neq I} A^{-1}(P)$ is dense in $H$ if and only if there exists an operator $B$ in $A \lg N$ such that $A A^{*}=B B^{*}$. We first prove some preliminary results.

Lemma 5. Let $N$ be a nest on $H$ and $A$ an operator in $B(H)$. The following are equivalent:

(a) The set $\bigcup_{P \in N, P \neq 0}\left(A^{*}\right)^{-1}\left(P^{\perp}\right)$ is dense in $H$.

(b) $\bigcap_{P \in N, P \neq 0} \overline{r(A P)}=\{0\}$.

Proof. We have that $\left(A^{*}\right)^{-1}\left(P^{\perp}\right)=\left\{x \in H: A^{*} x \in P^{\perp}\right\}=\left\{x \in H: P^{\perp} A^{*} x=A^{*} x\right\}$ $=\operatorname{Ker}\left(P A^{*}\right)=\overline{r(A P)}^{\perp}$ and $\bigcup_{P \in N, P \neq 0} \overline{r(A P)}{ }^{\perp}$ is dense in $\left(\bigcap_{P \in N, P \neq 0} \overline{r(A P)}\right)^{\perp}$. 
Proposition 6. Let $N$ be a nest on $H$ and $A$ an operator in $B(H)$.

(a) Suppose that the set $\bigcup_{P \in N, P \neq I} A^{-1}(P)$ is dense in $H$. Then $r(A)$ is $N$ proper.

(b) Suppose that $r(A)$ is $N$-proper and closed. Then the set $\bigcup_{P \in N, P \neq I} A^{-1}(P)$ is dense in $H$.

Proof. (a) The set $A\left(\bigcup_{P \in N, P \neq I} A^{-1}(P)\right)$ is contained in $[r(A) \cap P: P \in N, P \neq I]$ and is dense in $r(A)$.

(b) The restriction of $A$ to $\operatorname{coker} A$ is an isomorphism from coker $A$ onto $r(A)$. Hence $\left(\bigcup_{P \in N, P \neq I} A^{-1}(P)\right) \cap \operatorname{coker} A=A^{-1}\left(\bigcup_{P \in N, P \neq I} P\right) \cap \operatorname{coker} A$ is dense in coker $A$. Therefore $\bigcup_{P \in N, P \neq I} A^{-1}(P)$ is dense in $H$.

Proposition 7. Let $N$ be a nest on $H$ and $A$ an operator in $B(H)$.

(a) Suppose that $\bigcap_{P \in N, P \neq 0} \overline{r(A P)}=\{0\}$. Then coker $A$ is $N^{\perp}$-proper.

(b) Suppose that coker $A$ is $N^{\perp}$-proper and $r(A)$ is closed. Then $\bigcap_{P \in N, P \neq 0} \overline{r(A P)}$ $=\{0\}$.

Proof. (a) It follows from Lemma 5 that $\bigcup_{P \in N, P \neq 0}\left(A^{*}\right)^{-1}\left(P^{\perp}\right)$ is dense in $H$. It follows from Proposition 6 that $r\left(A^{*}\right)$ is $N^{\perp}$-proper. Since the closure of an $N^{\perp}$. proper subspace is an $N^{\perp}$-proper subspace we conclude that coker $A$ is $N^{\perp}$-proper.

(b) It follows from [2, Ch. VI, Th. 1.10] that $r\left(A^{*}\right)$ is closed. Hence $r\left(A^{*}\right)=$ coker $A$. It follows from Proposition 6 that $\bigcup_{P \in N, P \neq 0}\left(A^{*}\right)^{-1}\left(P^{\perp}\right)$ is dense in $H$. Therefore from Lemma 5 we conclude that $\bigcap_{P \in N, P \neq 0} \overline{r(A P)}=\{0\}$.

\section{FACTORISATION}

In this section we prove our main results and give some applications.

Theorem 8. Let $N$ be a continuous nest and $A$ an operator in $B(H)$. The following are equivalent:

(a) There exists an operator $B$ in $\operatorname{Alg} N$ such that $A A^{*}=B B^{*}$.

(b) The set $\bigcup_{P \in N, P \neq I} A^{-1}(P)$ is dense in $H$.

Proof. Assume (a) holds. In order to prove (b) it is enough to prove that the set $\bigcup_{P \in N, P \neq I}\left(A^{-1}(P) \cap \operatorname{coker} A\right)$ is dense in coker $A$. Using polar decomposition one can see that there exists a partial isometry $U$ with domain $\operatorname{coker} A$ and range $\operatorname{coker} B$ such that $A=B U$. We put $R=\overline{\left[\bigcup_{P \in N, P \neq I}\left(A^{-1}(P) \cap \operatorname{coker} A\right)\right]}$ and $M=$ coker $A \ominus R$. We will show that $M=\{0\}$. Take $m$ in $M$ and $P$ in $N, P \neq I$. Since $r(A)=r(B)\left(\left[5\right.\right.$, Th. 1]), we have $B P U m=A x_{P}$ for some $x_{P}$ in $\operatorname{coker} A$. Since $B P U m$ is in $P, x_{P}$ is in $A^{-1}(P) \cap \operatorname{coker} A$ and hence in $R$. We have $B P U m=$ $A x_{P}=B U x_{P}$ and so $P U m-U x_{P}$ is in ker $B$. We have $P U m=P U m-U x_{P}+U x_{P}$ which belongs to ker $B \oplus U R$. Note that the decomposition $H=\operatorname{ker} B \oplus U R \oplus U M$ is orthogonal. Therefore $U m=\lim _{P \in N, P \neq I, P \rightarrow I} P U m$ is in $(\operatorname{ker} B \oplus U R) \cap U M=\{0\}$. We conclude that $m=0$.

Assume (b) holds. It is then clear that the set $\bigcup_{P \in N, P \neq I}\left(A^{-1}(P) \cap \operatorname{coker} A\right)$ is dense in $\operatorname{coker} A$. Take a sequence $\left\{P_{n}\right\}_{n=0}^{\infty}$ of elements of $N$ such that: $P_{0}=0$, $P_{n+1}>P_{n}, P_{n} \neq I$ for every $n$ and $P_{n}$ converges strongly to $I$. We set: $R_{1}=$ $A^{-1}\left(P_{1}\right) \cap \operatorname{coker} A, R_{n}=\left(A^{-1}\left(P_{n}\right) \cap \operatorname{coker} A\right) \ominus R_{n-1}$ for $n>1$.

It is clear that $R_{n}$ is orthogonal to $R_{m}$ for $n \neq m$ and that $R_{n}$ is contained in $\operatorname{coker} A$ for every $n$. We show that $\operatorname{coker} A=\sum_{n=1}^{\infty} \oplus R_{n}$. Take $y$ in $\operatorname{coker} A$. If $y$ is orthogonal to $\sum_{n=1}^{\infty} \oplus R_{n}$, then $y$ is orthogonal to $A^{-1}\left(P_{n}\right) \cap \operatorname{coker} A$ for every 
$n$; hence $y$ is orthogonal to $\left(\bigcup_{n=1}^{\infty}\left(A^{-1}\left(P_{n}\right) \cap \operatorname{coker} A\right)\right)$. Since $\left(\bigcup_{n=1}^{\infty}\left(A^{-1}\left(P_{n}\right) \cap\right.\right.$ $\operatorname{coker} A)$ ) is dense in coker $A, y=0$, and so $\operatorname{coker} A=\sum_{n=1}^{\infty} \oplus R_{n}$.

Consider for $n \geq 1$ a partial isometry $V_{n}$ with domain contained in $\left(P_{n+1}-P_{n}\right) H$ and range $R_{n}$. Put $V=\sum_{n=1}^{\infty} \oplus V_{n}$. Then $V$ is a partial isometry with range $\operatorname{coker} A$. Note that $A=A V V^{*}$. We show that $A V$ belongs to $\operatorname{Alg} N$. Let $P$ be in $N$ and $x$ be a vector in $P$. We show that $A V x$ is in $P$. If $P \leq P_{1}$ we have $A V x=0$. If $P>P_{1}$ there exists $m \geq 1$ such that $P_{m}<P \leq P_{m+1}$. Then $A V x=A\left(\sum_{n=1}^{m} \oplus V_{n}\right) x$ and $\left(\sum_{n=1}^{m} \oplus V_{n}\right) x$ is contained in $\left(\sum_{n=1}^{m} \oplus R_{n}\right)$. Therefore $A V x$ is in $A\left(\sum_{n=1}^{m} \oplus R_{n}\right)$ which is contained in $P_{m}$. Since $P_{m}<P$ we conclude that $A V x$ is in $P$.

Put $B=A V$. Then $B B^{*}=A V V^{*} A^{*}=A A^{*}$ and $B$ is in $\operatorname{Alg} N$.

Remark. Theorem 8 remains true under the weaker assumption that $N$ is a nest which satisfies $H=\overline{\left[\bigcup_{Q<H} Q\right]}$.

Let $N$ be a continuous nest. We give an example of an operator with $N$-proper range which does not satisfy condition (b) of Theorem 8 .

Example 9. Let $N$ be a continuous nest. Take a sequence $\left\{P_{n}\right\}_{n=0}^{\infty}$ of elements of $N$ such that:

$$
P_{0}=0, \quad P_{n+1}>P_{n}, \quad P_{n} \neq I \quad \text { for every } n \text { and } P_{n} \text { converges strongly to } I .
$$

For each $n$ consider a vector $e_{n}$ of norm 1 and such that $\left(P_{n+1}-P_{n}\right) e_{n}=e_{n}$. Put $y=\sum_{i=1}^{\infty} n^{-1} e_{n}$. Let $A$ be the operator defined by: $A e_{n}=n^{-1} e_{n}$ for $n \geq 1$, $A e_{0}=y$ and $A$ is 0 on $\left[e_{n}: n=0,1,2, \ldots\right]^{\perp}$. Then $r(A)$ is $N$-proper and it is easy to see that $A$ does not satisfy condition (b) of Theorem 8 . In fact, $e_{0}$ is orthogonal to $\bigcup_{P \in N, P \neq I} A^{-1}(P)$. So $A$ does not satisfy condition (a) of Theorem 8 .

Corollary 10. Let $N$ be a continuous nest and $A$ an operator in $B(H)$. The following are equivalent:

(a) There exists an operator $B$ in $\operatorname{Alg} N$ such that $A^{*} A=B^{*} B$.

(b) $\bigcap_{P \in N, P \neq 0} \overline{r(A P)}=\{0\}$.

Proof. There exists an operator $B$ in $\operatorname{Alg} N$ such that $A^{*} A=B^{*} B$ if and only if there exists an operator $C$ in $\operatorname{Alg} N^{\perp}$ such that $A^{*} A=C C^{*}$. The corollary follows now from Theorem 8 and Lemma 5 .

Corollary 11. Let $N$ be a continuous nest and $A$ an operator in $B(H)$. Suppose $A$ is onto (resp. one-to-one and $r(A)$ is closed). Then there exists an operator $B$ in $\operatorname{Alg} N$ such that $A A^{*}=B B^{*}$ (resp. $\left.A^{*} A=B^{*} B\right)$.

Proof. It follows from Proposition 6 and Theorem 8 (resp. from Proposition 7 and Corollary 10).

Corollary 12. Let $N$ be a continuous nest and $Q$ a projection in $B(H)$. Then there exists an operator $B$ in $\operatorname{Alg} N$ such that $Q=B B^{*}\left(\right.$ resp. $\left.Q=B^{*} B\right)$ if and only if $Q H$ is $N$-proper (resp. $N^{\perp}$-proper).

Proof. It follows from Proposition 6 and Theorem 8 (resp. from Proposition 7 and Corollary 10).

The following corollary answers a question posed by Shields in [13]. 
Corollary 13. Let $N$ be a continuous nest and $A$ a positive operator in $B(H)$. Assume there exists an operator $B$ in $\operatorname{Alg} N$ such that $A^{2}=B^{*} B$. Then there exists an operator $C$ in $\operatorname{Alg} N$ such that $A=C^{*} C$.

Proof. We have to show that if $\bigcap_{P \in N, P \neq 0} \overline{r(A P)}=\{0\}$, then $\bigcap_{P \in N, P \neq 0} \overline{r\left(A^{1 / 2} P\right)}$ $=\{0\}$. Let $y$ be in $\bigcap_{P \in N, P \neq 0} \overline{r\left(A^{1 / 2} P\right)}$. Then $A^{1 / 2} y$ is in $\bigcap_{P \in N, P \neq 0} \overline{r(A P)}=\{0\}$; hence $A^{1 / 2} y=0$. So $y$ is in Ker $A^{1 / 2}$. Since $y$ is also in $\overline{r\left(A^{1 / 2}\right)}$ we see that $y=0$.

We will characterise now the operators that satisfy condition (a) of Theorem 8 (resp. condition (a) of Corollary 10) for every continuous nest.

Proposition 14. Let $V$ be an operator range. Assume $V$ is not of co-finite dimension in $H$. Then there exists a continuous nest $N$ in $H$ such that $V \cap P=\{0\}$ for every $P$ in $N, P \neq I$.

Proof. (i) We first show that there exists a non-closed operator range $W$ which contains $V$. We will use the following fact: If $V_{1}, V_{2}$ are operator ranges, then $V_{1}+V_{2}$ is an operator range [7, Ch. I, 1]. If $V$ is closed we consider an operator range $U$ which is non-closed and is contained in $V^{\perp}$. We set $W=V+U$. Then $W$ is an operator range which is non-closed and contains $V$.

(ii) It follows from (i) above that we may assume that $V$ is non-closed. An operator range $R$ is called of type $J_{S}$ (Dixmier's notation) if it is dense and there exists a sequence $\left\{H_{n}\right\}_{n=0}^{\infty}$ of closed mutually orthogonal infinite dimensional subspaces of $H$ such that $R=\left\{\sum_{n=0}^{\infty} x_{n}: x_{n} \in H_{n}\right.$ and $\left.\sum_{n=0}^{\infty}\left(2^{n}\left\|x_{n}\right\|\right)^{2}<\infty\right\}$. It is shown in the proof of Theorem 3.6 in [6] that any non-closed operator range is contained in an operator range of type $J_{S}$. It follows that there exists an operator range $S$ of type $J_{S}$ such that $V \subset S$. It follows from Theorem 3.6 in [6] that there exists a unitary operator $U$ on $H$ such that $U S \cap S=\{0\}$. We conclude that there exists an operator range $T$ of type $J_{S}$ such that $V \cap T=\{0\}$. Now it is easy to see that there exists a continuous nest $N$ in $H$ such that $P \subset T$ for every $P$ in $N, P \neq I$. It follows that $P \cap V=\{0\}$ for every $P$ in $N, P \neq I$.

Theorem 15. Let $A$ be an operator in $B(H)$.

(a) There exists for every continuous nest $N$ an operator $B_{N}$ in $\operatorname{Alg} N$ satisfying $A A^{*}=B_{N} B_{N}^{*}$ if and only if $A$ is a right Fredholm operator.

(b) There exists for every continuous nest $N$ an operator $B_{N}$ in $\operatorname{Alg} N$ satisfying $A^{*} A=B_{N}^{*} B_{N}$ if and only if $A$ is a left Fredholm operator.

Proof. (a) Assume that for every continuous nest $N$ there exists an operator $B_{N}$ in $\operatorname{Alg} N$ satisfying $A A^{*}=B_{N} B_{N}^{*}$. It follows from Theorem 8 and Proposition 6 that $r(A)$ is $N$-proper for very continuous nest $N$. Proposition 14 implies that $r(A)$ is of co-finite dimension in $H$. If the range of an operator is of co-finite dimension, then it is closed [4, Prop. 3.7]. Therefore $A$ is a right Fredholm operator. Assume now that $A$ is a right Fredholm operator. Then $r(A)$ is closed and of co-finite dimension in $H$. By Proposition 4, $r(A)$ is $N$-proper for every continuous nest $N$. It follows then from Proposition 6 and Theorem 8 that for every continuous nest $N$ there exists an operator $B_{N}$ in $\operatorname{Alg} N$ satisfying $A A^{*}=B_{N} B_{N}^{*}$.

(b) Consider the following properties of an operator $A$ :

(i) There exists for every continuous nest $N$ an operator $B_{N}$ in $\operatorname{Alg} N$ satisfying $A A^{*}=B_{N} B_{N}^{*}$. 
(ii) There exists for every continuous nest $N$ an operator $B_{N}$ in $\operatorname{Alg} N$ satisfying $A^{*} A=B_{N}^{*} B_{N}$.

Since a nest $N$ is continuous if and only if the nest $N^{\perp}$ is continuous we see that an operator $A$ has property (i) if and only if the operator $A^{*}$ has property (ii). The assertion follows now from (a).

\section{ADDED IN PROOF}

After this work was submitted a paper of G. T. Adams, J. Froelich, P. J. McGuire, and V. I. Paulsen entitled Analytic reproducing kernels and factorisation, Indiana Univ. Math. J. 43 (1994), came to our attention. Condition (b) of our Theorem 8 is essentially the same with the density condition given in Theorem 3.1 of this paper in a different but related context.

\section{REFERENCES}

1. W. B. Arveson, Interpolation problems in nest algebras, J. Funct. Anal. 20 (1975), 208-233. MR 52:3979

2. J. B. Conway, A course in functional analysis, Springer-Verlag, 1985. MR 86h:46001

3. K. R. Davidson, Nest algebras, Pitman Research Notes in Mathematics Series, 191 (1988). MR 90f: 47062

4. J. Dixmier, Etude sur les variétés et les opérateurs de Julia, Bull. Soc. Math. France $\mathbf{7 7}$ (1949), 11-101. MR 11:369f

5. R. G. Douglas, On majorization, factorization and range inclusion of operators in Hilbert space, Proc. Amer. Math. Soc. 17 (1966), 413-416. MR 34:3315

6. P. A. Fillmore and J. P. Williams, On operator ranges, Advances in Math. 7 (1971), 254-281. MR 45:2518

7. C. Foias, Invariant para-closed subspaces, Indiana Univ. Math. J. 21 (1972), 887-906. MR 53:3734

8. I.C. Gohberg and M. G. Krein, Theory and applications of Volterra operators in Hilbert space, Transl. Math. Monographs, 24 (1970), AMS. MR 41:9041

9. D. R. Larson, Nest algebras and similarity transformations, Ann. of Math. 121 (1985), 409427. MR 86j:47061

10. D. R. Pitts, Factorization problems for nests: Factorization methods and characterizations of the universal factorization property, J. Funct. Ana. 79 (1988), 57-90. MR 90a:46160

11. S. C. Power, Nuclear operators in nest algebras, J. Operator Theory 10 (1983), 337-352. MR 85b: 47028

12. S. C. Power, Factorisation in analytic operator algebras, J. Funct. Anal. 67 (1986), 413-432. MR 87k:47040

13. A. L. Shields, An analogue of a Hardy-Littlewood-Fejer inequality for upper triangular trace class operators, Math. Z. 182 (1983), 473-484. MR 85c:47022

Department of Mathematics, University of the Aegean, Karlovassi 83200, Greece

Department of Mathematics, East Carolina University, Greenville, North Carolina 27858 